\title{
Design and research of measuring system of clamping force of hemostats
}

\author{
Huifang Wang, Shuyi Wang, Ying Zhou, Jie Tan
}

School of Medical Instrument and Food Engineering, University of Shanghai for Science and Technology, Shanghai, China. Email: fengerimissyou@163.com

Received 15 July 2010; revised 25 July 2010; accepted 28 July 2010.

\begin{abstract}
Reliability of medical instruments such as hemostats is extremely important because these instruments are used in patients who are in critical condition. Clamping force of hemostats, as an important parameter of hemostats, should be detected. However, it could not be tested directly. In order to test it, a testing system has been put forward. The system is comprised of sensor, acquisition card, and three-way tap and so on. This system is controlled by a computer. The testing system has been proved to be effective in testing the clamping force of hemostats.
\end{abstract}

Keywords: Hemostat; Clamping Force; Measuring System

\section{INTRODUCTION}

A hemostat is a vital surgical tool used in almost any surgical procedure, usually to control bleeding. Hemostats belong to a group of instruments that pivots (similar to scissors, includes needle holders, tissue holders and various clamps), and where the structure of the tip determines their function. Hemostats have handles that can be held in place by the locking mechanism. The locking mechanism is typically a series of interlocking teeth, a few on each handle that allow the user to adjust the clamping tension of the pliers [1]. However, pressure values are different regarding to different hemostats.

Medical equipment manufacturers can produce many kinds of hemostats, but inspection standard and inspection method of hemostats remain be not researched and improved, especially clamping force of hemostats remains cannot be tested directly or indirectly. However, clinicians or inspection personnel both hope to know the quantitative pressure.

In both at home and abroad, clamp pressure measureement has been fixed in the field of auto and robots. In brake-by-wire systems of auto, central controllers requ- ire accurate information about the clamp force between the brake pad and the disc as a function of pad displacement, which is usually denoted as the characteristic curve of the caliper. Due to aging, temperature, and other environmental variations, caliper characteristic curves vary with time. Therefore, automatic caliper calibration in real-time is vital for high-performance braking action and vehicle safety [2]. In robots, firstly design machine gripper according to the features of the object of holding. Secondly analyzes forces of all parts of machine gripper. At last, derivate clamp pressure force according to the principle of torque. In these machine grippers some are two-finger translation grippers [3] and some are curved arm type hydraulic grab with four bar linkage [4]. There are hardly some studies in clamp pressure measurement of hemostats.

Existing pressure sensors in shape and size and fatigue resistance level are unable to satisfy the special needs of these medicinal hemostats, so direct measurement of clamping force of hemostats needs design special sensor system. However the sensor system has not been worked out so far according to my study. So some attentions have been focused on finding an indirect measurement method.

An indirect measurement method is proposed in this paper. Through testing pressure changes of a clamping catheter filled with liquid, clamping force of hemostats can be reflected indirectly.

\section{METHODS AND APPARATUS}

\subsection{Description of Apparatus}

Measuring system of clamping force of hemostats is designed which is shown in Figure 1.

The testing system can be divided into two parts according to their different functions.

Part 1: In this part, hardware principle of the test system is provided. Functions of each component as follows:

1) Injector: Eliminate air and fill liquid in the whole system. 


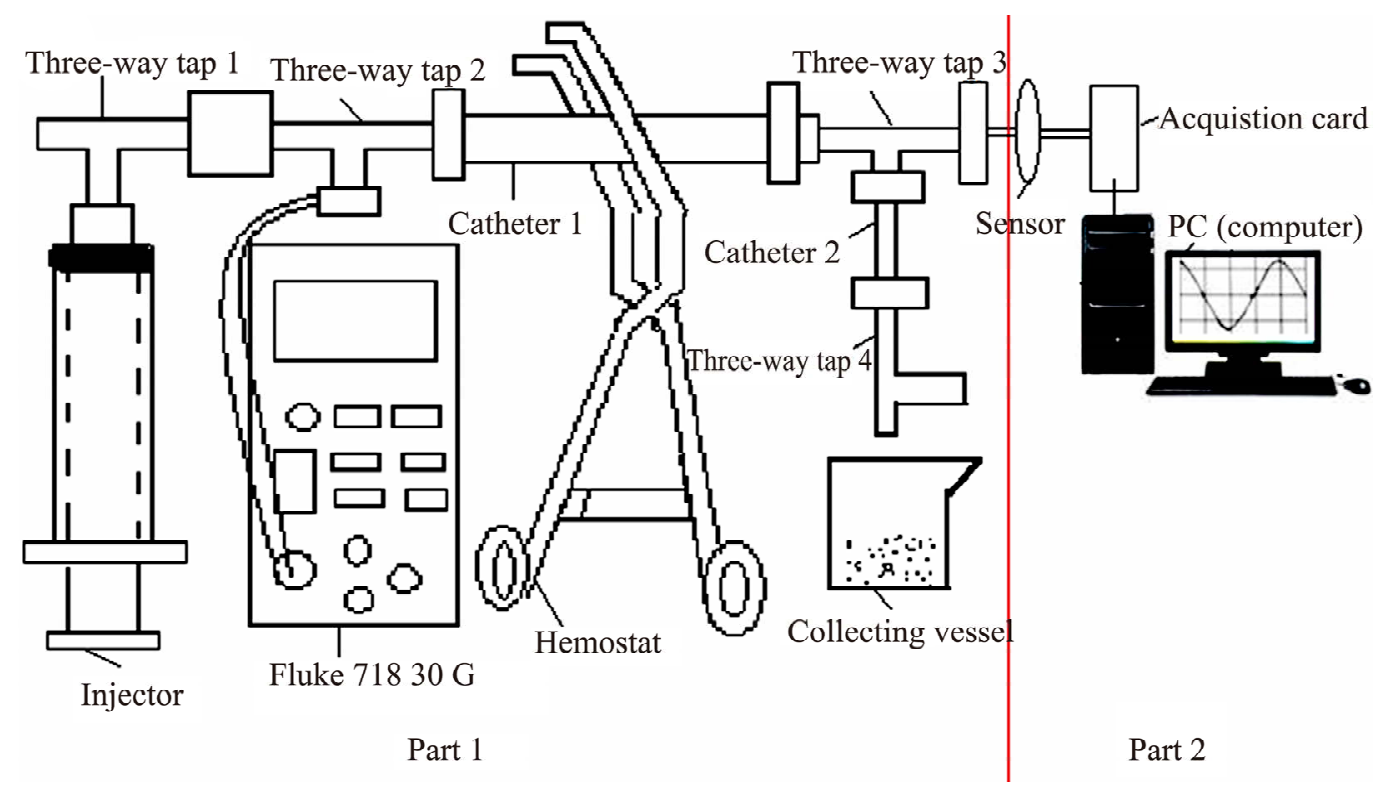

Figure 1. Structure of testing system of clamping force of hemostats.

2) Three-way tap 1: Make the whole system separate from air.

3) Three-way tap 2: Used for the switch of calibration and testing of pressure.

4) Fluke 718 30G: Provide pressure to the system.

5) Catheter 1(Oxygen tubes) and hemostat (XAB010 made in Shanghai Medical Instruments (Group) Corp., Ltd. Surgical Instruments Factory): Clamp catheter one using a hemostat.

6) Three-way tap 3: Test whether there is air or not, and make sure there is no air in the whole system.

7) Three-way tap 4: Make liquid inject easily into the whole system.

8) Collecting vessel: Collect the liquid while injecting liquid into the system.

9) Catheter 2: Any catheter can be used.

Part 2: In this part, the interface between PC running under Windows XP and sensor was realized through an RS-232 connection. The sampling rate is controlled by PC and software, which is based on Visual $\mathrm{C}++6.0$ platform. The signal coming from sensor is obtained by acquisition card, and then is transmitted to computer. Last data signals could be displayed on monitor.

\subsection{Measurements}

The measurement is carried out using a test solution of ISO class III water for medical use, under normal condition $\left(25^{\circ} \mathrm{C} \pm 2{ }^{\circ} \mathrm{C}, 58 \% \pm 5 \% \mathrm{RH}\right.$ (Relative humidity)). There are several steps to implement this apparatus:

First: Connect all the joints and check the testing system. The whole system should be filled with water in advance.
Second: Calibrate of the testing system. Pressure sensor is an important part in testing system, and the accuracy and precision of sensor are influenced by actual environment, such as temperature, relatively humidity. So to ensure the accuracy of the testing result, pressure calibration of the sensor and the whole testing system is necessary before testing.

Third: Test clamping force of hemostats. The real-time pressure values could be displayed in monitor.

\section{RESULTS}

In this testing system, pressure calibrator of Fluke-718 $30 \mathrm{G}$ which has $\pm 0.05 \%$ of range and resolution is 0.01 $\mathrm{kPa}$ was used, it can be used to calibrate pressure sensor whose range of pressure is $0-200 \mathrm{kPa}$ and the whole testing system [5].The unit of calibrator is $\mathrm{kPa}$ and computer display is $\mathrm{V}$ (voltage), and computer display is the value of pressure signal, which has been magnified and filtered.

Based on the relationship between calibrator value and computer display value, a line could be drawn. The relation between the display of computer and pressure calibrator is almost linear. The expression was calculated with least squares approximation and it is:

$$
y=55.531 * x-102.81
$$

where $x$ is the actual pressure obtained from an acquisition card and $y$ is the value of calibrator.

Oxygen tubes (GB15593-1995) were repetitive tested under the same condition, including temperature $\left(25^{\circ} \mathrm{C} \pm\right.$ $\left.2^{\circ} \mathrm{C}\right)$, relative humidity $(58 \% \pm 5 \%)$ and initial pressure (30 $\mathrm{kPa}$ ). In the testing system, a hemostat, which has 
pressure value (Unit: $\mathrm{kPa}$ )

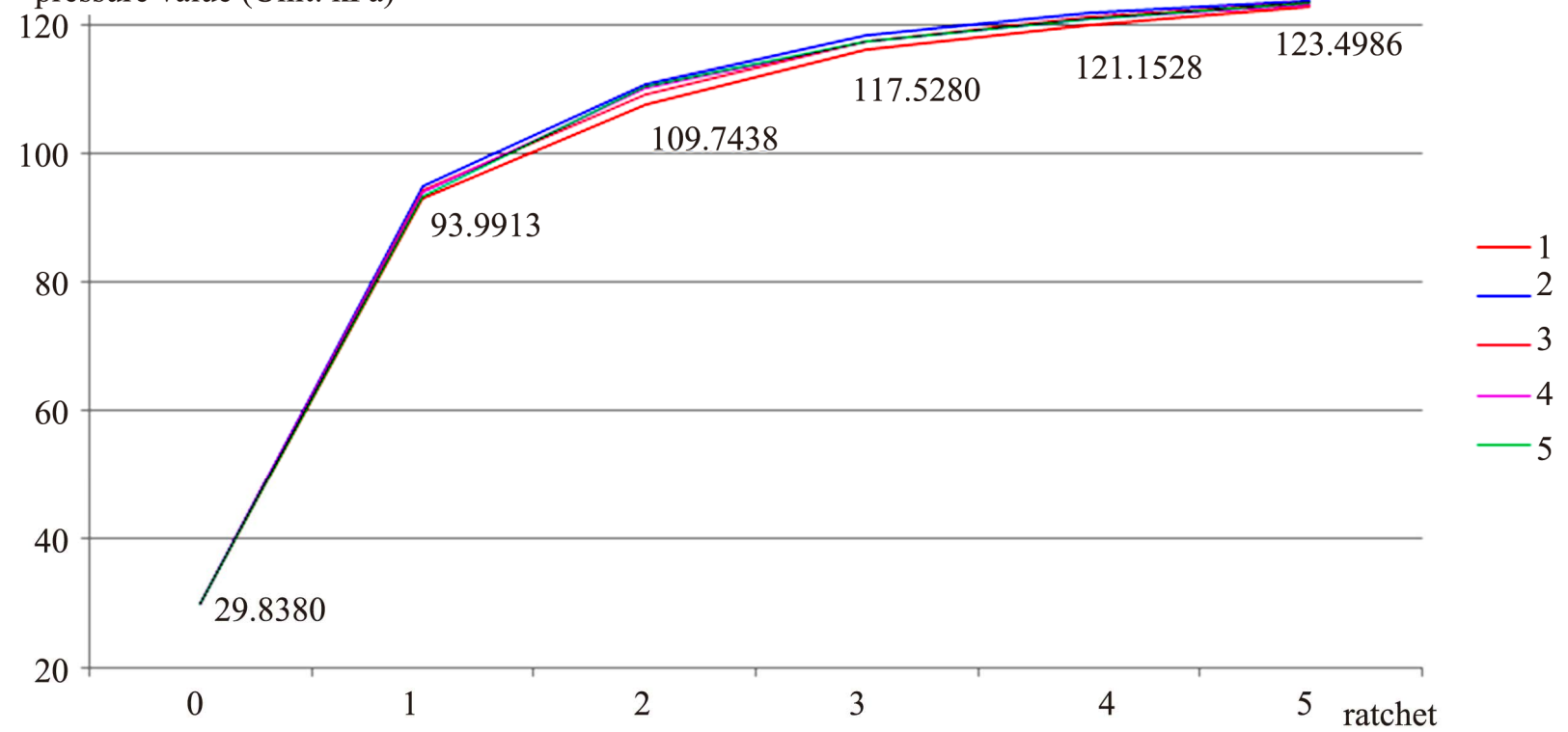

Figure 2. Pressure values of different ratchets of hemostats.

five ratchets [6], was used. In Figure 2, 0 represents the hemostat was not be used,1,2,3,4, and 5 represents respectively the first, second, third, fourth and fifth ratchet was used. Pressure values of clamping force of hemostats are shown in Figure 2. The average values of the pressure of each ratchet are signed in the Figure 2. The lines of 5 different colors represent that the repetitive tests were performed 5 times.

\section{DISCUSSION AND CONCLUSIONS}

The drawing lines in Figure 2 are tested results of a hemostat and these tests are repeated implemented under normal environment. From these drawing lines above, the average value of the pressure of the first ratchet is $93.9913 \mathrm{kPa}$, the second ratchet is $109.7438 \mathrm{kPa}$, the third ratchet is $117.5280 \mathrm{kPa}$, the fourth ratchet is 121.1528 $\mathrm{kPa}$ and the fifth ratchet is $123.4986 \mathrm{kPa}$. Some results can also be obtained: when the second ratchet is used, the error which is less than $3 \mathrm{kPa}$ is maximal, and when some ratchets are used, the data is nearly same. So to some extent clamping force of hemostats can be reflected indirectly. Thus in turn the testing measure and system is proved to be corrected.

The sensor is influenced by environment, especially by temperature. In order to ensure the accuracy of the test, calibration process should be implemented before performance, even in stable environment, calibration should be operated periodically.

In this study the measuring system of clamping force of hemostats is designed. The results of measuring system of clamping force of hemostats have verified that this method is efficient. In the testing, after doing an experiment, if another repeated experiment needs to be done, experimenters need to wait for a period of time, at least 4 minutes, to make catheters restore deformation. The future research is concerned with these following topics:

1) The system is influenced by environment, such as temperature, relative humidity, so more tests should be implemented to come to a much better relation between system and environment, which can be a reference for further tests.

2) In the past experiments only one kind of catheter and only one hemostat was used. As we all know, there are many kinds of catheters and hemostats. And if the catheter is too soft and the pressure of the hemostat is too high, the catheter is destroyed permanently and ratchets of hemostats is useless. But if the catheter is too rigid and the pressure of the hemostats is too low, there is not tiny deformation after clamping catheters using hemostats. The theory applies to clamp blood vessels using hemostats. If vascular wall is too thin, but the pressure of hemostats is too high, the blood vessels will be ruptured. However if vascular wall is too thick and the pressure of hemostats is too low, bleed will still flow from blood vessels. Thus patients are very dan- gerous. So it needs to further study to which hemostat matching to which catheter.

\section{ACKNOWLEDGEMENTS}

The research described in this paper is being supported by Initial Funding for Doctor of University of Shanghai for Science and Technology and supported by Funded Projects of Key Discipline of Shanghai, Project Number: T0502. 


\section{REFERENCES}

[1] http://www.answers.com/topic/hemostat

[2] Hoseinnezhad, R., Bab-Hadiashar, A. and Rocco, T. (2008) Real-time clamp force measurement in electromechanical brake calipers. IEEE Vehicular Technology Society, 57(2), 770-777.

[3] Su, Z.H. and Kong, Q.Z. (2008) Design of two- finger translation gripper of articulated robot. Mechanical Engineering \& Automation, (5), 80-84.

[4] Ma, Z.S., Bian, X.L. and Chu, X.J. (2008) Design and performance simulation for grab on emergency engineering robot. Engineering Machinery, 39(7), 25-28.

[5] Pressure Calibrator 718 Product.

[6] Zhang, H.Y., Li, B. and Xu, Z.T. (2008) Maintenance and inspection of surgical instrument. Operation \& Maintenance, 29(9), 126. 